\title{
Analysis of the time-resolved magneto-optical Kerr effect for ultrafast magnetization dynamics in ferromagnetic thin films
}

\author{
I Razdolski ${ }^{1}$, A Alekhin ${ }^{1} \ddagger$, U Martens ${ }^{2}$, D Bürstel $^{3}$, D Diesing ${ }^{3}$, \\ M Münzenberg ${ }^{2}$, U Bovensiepen ${ }^{4}$ and A Melnikov ${ }^{1,5}$ \\ ${ }^{1}$ Fritz Haber Institute of Max Planck Society, Faradayweg 4-6, 14195 Berlin, \\ Germany \\ 2 Ernst Moritz Arndt University, Institute for Physics, Felix-Hausdorff-Str. 6,17489 \\ Greifswald, Germany \\ ${ }^{3}$ Faculty of Chemistry, University of Duisburg-Essen, Universitätsstr. 5, 45141 \\ Essen, Germany \\ 4 Faculty of Physics and Center for Nanointegration (CENIDE), University of \\ Duisburg-Essen, Lotharstraße 1, 47057 Duisburg, Germany \\ ${ }^{5}$ Faculty of Physics, Martin Luther University of Halle-Wittenberg, \\ Von-Dackelmann-Platz 3, 06120 Halle, Germany \\ E-mail: razdolski@fhi-berlin.mpg.de
}

9 February 2017

\begin{abstract}
We discuss fundamental aspects of laser-induced ultrafast demagnetization probed by the time-resolved magneto-optical Kerr effect (MOKE). Studying thin Fe films on $\mathrm{MgO}$ substrate in the absence of electronic transport, we demonstrate how to disentangle pump-induced variations of magnetization and magneto-optical coefficients. We provide a mathematical formalism for retrieving genuine laser-induced magnetization dynamics and discuss its applicability in real experimental situations. We further stress the importance of temporal resolution achieved in the experiments and argue that measurements of both time-resolved MOKE rotation and ellipticity are needed for the correct assessment of magnetization dynamics on sub-picosecond timescales. The framework developed here sheds light onto the details of the timeresolved MOKE technique and contributes to the understanding of the interplay between ultrafast laser-induced optical and magnetic effects.
\end{abstract}

PACS numbers: 78.20.Ls, 75.78.Jp, 72.25.Ba

Keywords: magnetization dynamics, magneto-optical Kerr effect, electronic transport, hot electrons, ultrafast demagnetization

$\ddagger$ Present address: IMMM UMR CNRS 6283, Universite du Maine, Avenue Messiaen, 72085 Le Mans, France. 


\section{Introduction}

Shortly after the first report of ultrafast, laser-induced demagnetization 20 years ago on nickel by Beaurepaire et al. [1] it was discussed whether a femtosecond timeresolved pump-probe experiment detecting the magneto-optical Kerr effect (MOKE) is a reliable tool to probe ultrafast photo-induced changes of the magnetization $M$. Few years later Koopmans et al. considered variations of the magneto-optical coefficients on the ultrafast timescale as a contribution to the observed transient differences in the real and imaginary part of the complex MOKE (Kerr rotation and ellipticity) [2]. Significantly different observations were then reported by Bigot and co-workers [3]. Interestingly, the investigated samples in these two papers used metallic and insulating substrates, respectively. This difference hinted early on to an essential influence of transport contributions in ultrafast magnetization dynamics. Addressing this issue explicitly, a theoretical study by Battiato et al. [4] demonstrated a good agreement with experimental observations $[5,6,7,8]$.

Nowadays it is widely accepted that, first, the phenomenon of sub-picosecond demagnetization of ferromagnets (FM) is real. Corroborating evidence obtained by employing further experimental methods, such as time- ans spin-resolved photoemission $[9,10,11,12]$, magnetic linear dichroism in photoemission [13], and X-ray magnetic circular dichroism $[14,15,16,17,18]$, represented an essential step for reaching this conclusion. It was, furthermore, rewarding to achieve agreement between experimental findings and theoretical modeling considering Elliott-Yafet spin flip scattering [19], although this theory has been challenged by others [20]. Moreover, various theoretical results based on purely electronic effects mediated by spin-orbit coupling were reported $[21,22,23]$. Second, it is by now clear that an unambiguous analysis of femtosecond magneto-optical results is non-trivial, owing to the sizeable transient changes of the electron population $[24,25,26]$. Later it was suggested that further insights into magnetization dynamics can be obtained when using different pump and probe optical wavelengths for time-resolved MOKE [11, 19], employing non-linear optical technique to address different electronic states with the pump and probe beams [27] or performing a detailed polarimetric analysis separating charge and spin contributions to time-resolved MOKE signals [28, 29]. Direct accounting for the respective impact of these effects on the transient magnetization as recently demonstrated for the different probing depth of the real and imaginary part of the complex MOKE [30] represents another perspective. This large amount of dedicated effort put into distinguishing spin and electron dynamics in MOKE transients as well as general popularity of the time-resolved MOKE technique has been motivated by its table-top availability and ultrashort temporal resolution, hardly reachable by other experimental methods. However, an explicit analysis of magnetooptical effects occurring due to electron repopulation after femtosecond laser excitation and means to correct the observed MOKE signals to reach the transient magnetization have not been developed so far.

In this Article, we present an overview of physical processes occurring during and 
immediately after femtosecond laser irradiation of a metallic FM. We compare transient MOKE data obtained on three different Fe samples and demonstrate that both FM film thickness and the substrate properties have a strong impact on the observable MOKE signals. We discuss in details how the effects of electronic repopulation make their way into the registered transient MOKE signals thus impeding correct determination of magnetization dynamics. In a special case of uniform excitation of a thin FM film on a dielectric $(\mathrm{MgO})$ substrate, thus excluding the possibility of electronic transport, we propose a way to retrieve the genuine laser-induced magnetization dynamics from the transient MOKE signals.

\section{Local and non-local magnetization dynamics}

The scope of this paper has its roots in considering a typical experiment on ultrafast laser-induced demagnetization of a metallic ferromagnetic (FM) film. Specifically, we consider a FM film of a few tens of nm thick, that is, considerably larger than the optical absorption depth $\delta \approx 15 \mathrm{~nm}$ in the visible range, thus resulting in a strongly inhomogeneous absorption profile across the FM film. Because femtosecond laser pulses deposit considerable amounts of energy, we further consider a metallic substrate as an effective heat sink. Here, laser irradiation results in the excitation of non-equilibrium hot carriers (HCs) which have spin-dependent, inhomogeneous spatial distribution both in the FM film and across the whole structure. Owing to the transfer of excitation into initially cold, non-excited spatial regions, the HCs dynamics becomes very complex $[31,32]$, involving both local and non-local physical processes (see Figure 1,a).

In what follows, we shall give an overview of the local and non-local HC dynamics. Femtosecond laser excitation locally repopulates empty electronic states with HCs. This repopulation continues as the strongly non-equilibrium distribution of HCs evolves with time [31], leading to the transient variations of the optical response of the sample. Furthermore, HCs in the FM exhibit spin-flip scattering which is often considered the backbone of ultrafast laser-induced demagnetization. This scattering is accompanied by a transfer of angular momentum from the spin system elsewhere, presumably to the lattice [33]. These two principle contributions to the local $\mathrm{HC}$ dynamics are further referred to as state-filling and demagnetization effects.

On the other hand, an inhomogeneous distribution of laser-excited HCs across the sample enables spin-polarised superdiffusive transport $[4,6,34,35]$. Besides spin injection into a conducting substrate, spin transport includes redistribution of the spin density within the FM film, which results in transient variations of the MOKE signal due to in-depth sensitivity of the latter $[36,37,30]$. On top of that, electronic transport is accompanied by the transfer of energy thus promoting heat-induced demagnetization in initially non-excited, cold parts of the sample, as discussed in [35] and termed "heat transport" in Figure 1. In a similar way, the non-local dynamics of HCs enables the state-filling transport contribution to the transient variations of the magnetooptical coefficients. It is thus seen that in general, both magnetic (related to the 


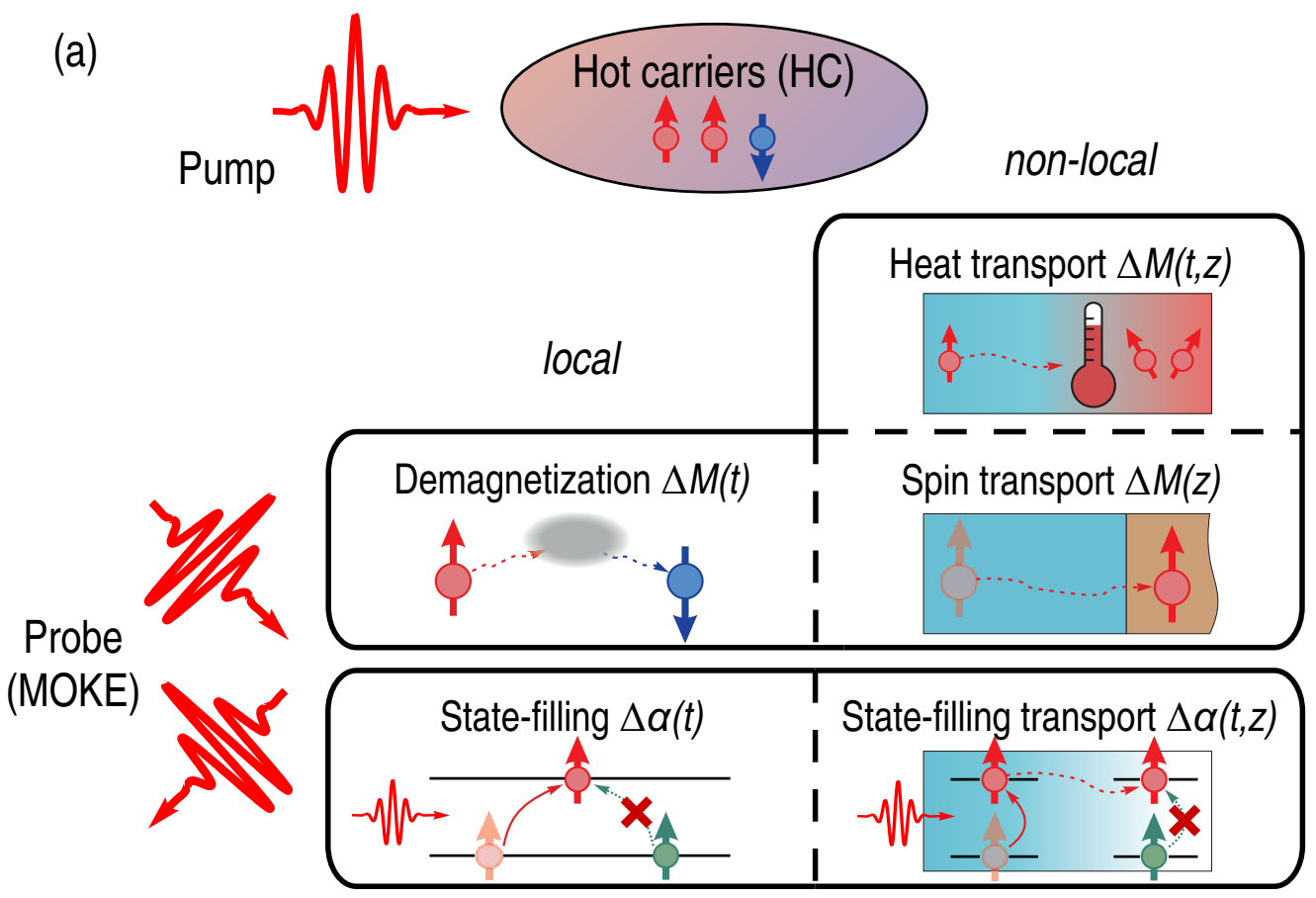

(b)

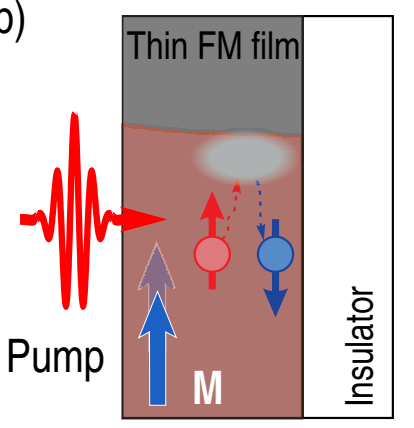

(c)

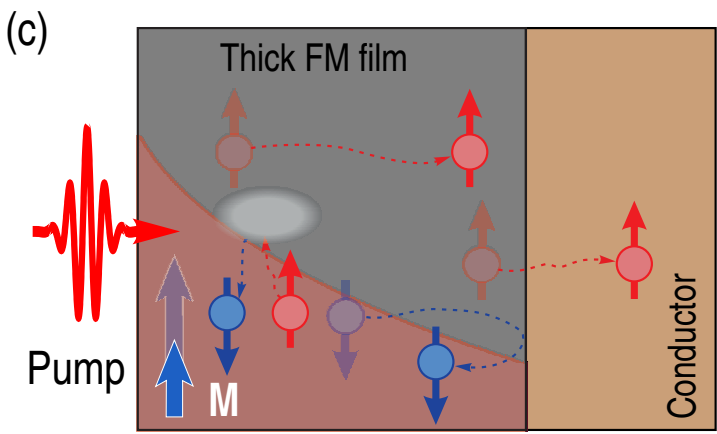

Figure 1. (a) Local and non-local electronic processes occurring upon femtosecond laser excitation of a ferromagnet leading to the emergence of hot non-equilibrium electrons. (b-c) Sketch of a femtosecond laser excitation of (b) a thin FM film on an insulating substrate, allowing for local processes only, and (c) thick FM film on a conducting substrate, where both local and non-local processes are present. The red shaded areas represent the in-depth profiles of the laser excitation.

real magnetization dynamics) and optical (caused by the variations of magneto-optical coefficients) contributions of the transient MOKE signal have local and non-local origins.

Summarizing these considerations, we note that an important step towards understanding the laser-induced demagnetization requires retrieving the real magnetization dynamics $M=M(t)$ in the absence of non-local contributions [8, 35]. To achieve this we engineered a system where (i) the excitation gradients are negligibly small (red shaded area in Figure 1,b) thus inhibiting the transport effects across the FM layer, and (ii) a dielectric substrate blocks the electronic transport out of the FM film (cf. Figure 1,b-c). 


\section{Transient changes of the magneto-optical coefficients}

Once the non-local effects are minimized, our further analysis aims at establishing a relation between the observable MOKE transients and magnetization dynamics. We consider the MOKE signal $\theta$ proportional to the magnetization $M: \theta=\alpha M[38,39]$, where $\alpha$ is the (effective) magneto-optical coefficient, and $\theta$ is either MOKE rotation or ellipticity. Following [40], in the experiments where time-resolved variations of the polarisation state of light $\Delta \psi$ are measured, it can be expanded into $\Delta \psi=\Delta \theta+\Delta b$, where $\Delta b$ represents even in magnetization pump-induced effects, including the nonmagnetic ones such as optical Kerr effect and others. To get access to magnetization dynamics, these even effects can be excluded by considering the difference of the datasets obtained at the two opposite directions of magnetization: $\Delta \theta=[\Delta \psi(+M)-$ $\Delta \psi(-M)] / 2$. After that, for sufficiently small absolute MOKE variations $\Delta \theta$ one gets:

$$
\Delta \theta=\alpha \cdot \Delta M+M \cdot \Delta \alpha,
$$

or, for relative variations $\delta \theta=\Delta \theta / \theta_{0}$ :

$$
\delta \theta=\delta M+\delta \alpha,
$$

Here $\theta_{0}=\alpha_{0} M_{0} \approx \alpha M$ is the corresponding static (equilibrium) MOKE signal, and $\delta M=\Delta M / M, \delta \alpha=\Delta \alpha / \alpha$.

It is thus seen that the laser-induced variations of magneto-optical coefficients (Eq. 2) are responsible for the observed difference in the transients of MOKE rotation and ellipticity $[2,3]$. Here we analyze this effect in details, arguing that understanding the nature of this contribution to the MOKE transients is crucial for drawing conclusions regarding magnetization dynamics. Magneto-optical coefficients are determined by matrix elements of the optically-active electronic transitions in the majority and minority sub-bands of a ferromagnet and by related densities of initial and final states. The population of these states depends on the electronic distribution and thus can be affected by the absorption of photons. Upon this absorption, a non-equilibrium distribution of electrons in the metal is created, which then decays towards an equilibrium at an elevated electronic temperature [25]. As we show below, modification of the electronic distribution leads to the variations of the magneto-optical coefficients thus altering the observable transient MOKE signals.

The possible mechanisms of the modification of magneto-optical coefficients due to electronic repopulation are illustrated in Figure 2,a-b. Firstly, we consider the effect of a direct optical excitation of primary electrons, which is particularly important in the case of equal pump and probe photon energies. In a simplified picture, upon a single photon absorption process an electron is created in the upper, previously empty state, and a hole appears at the initial electronic level. As such, until the excited state is relaxed, the variations of electronic population at both initial and final levels inevitably modify the optical response of the medium, including MOKE [24] (see Figure 2,a). Note that if the pump and probe photon energies are different, this mechanism can also be 

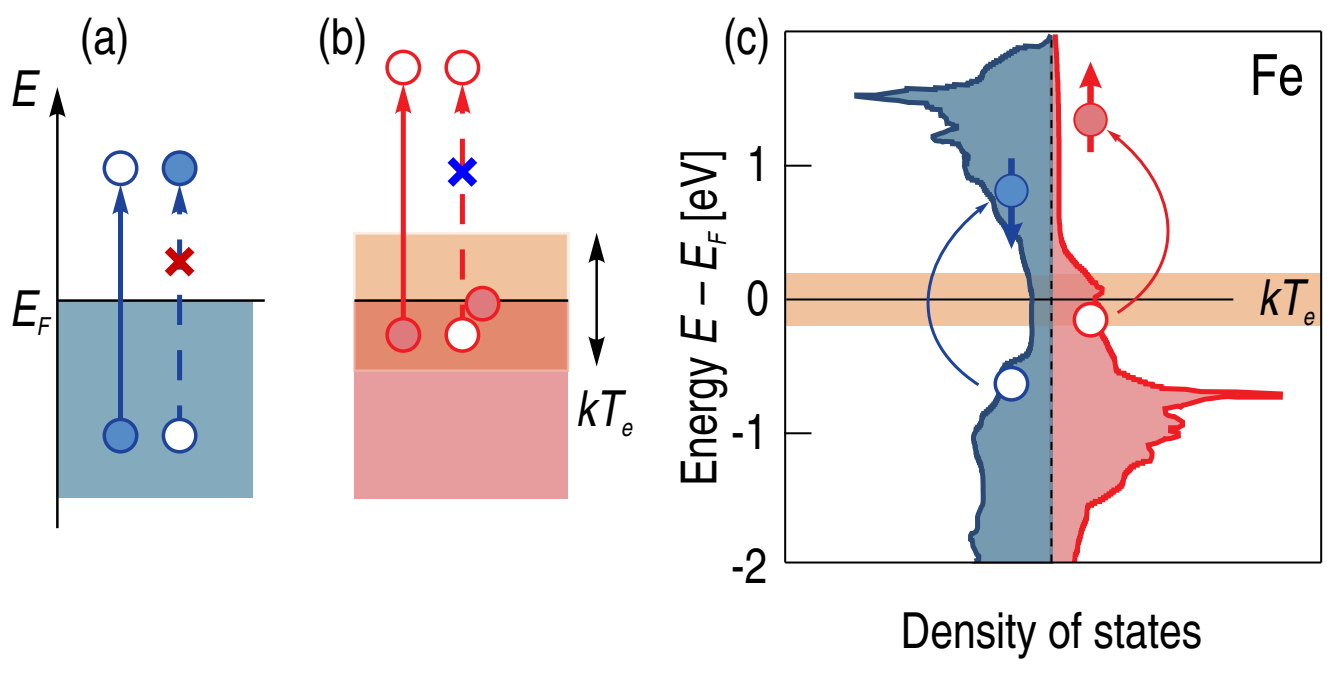

Figure 2. (a) Direct optical and (b) heating-induced state-filling effects as the two main mechanisms of electronic repopulation-induced variations of the optical response. Open and full circles show empty and filled electronic states, respectively, while solid and dashed lines with crosses indicate allowed and forbidden electronic transitions, respectively. (c) Density of states in Fe as redrawn from Ref. [41]. The arrows, empty and solid circles show predominant electronic transitions in both sub-bands if excited with $1.55 \mathrm{eV}$ photons. The shaded area illustrates the region of $E_{F} \pm k T_{e}$ at elevated electronic temperatures reached upon laser excitation.

important when either initial or final states of the pumped transitions participate in the probed transitions as well.

A more subtle yet important mechanism is illustrated in Figure 2,b. Consider an equilibrium (Fermi-Dirac) electronic distribution at a finite temperature $T_{e}$. Variations of $T_{e}$ will redistribute electrons in the $E_{F} \pm k T_{e}$ energy range $\left(E_{F}\right.$ is the Fermi energy) thus altering the probabilities of the corresponding electronic transitions. As such, if for a particular photon energy $\hbar \omega \gg k T_{e}$ electronic states from this range contribute to the optical response of a medium, the latter is modified by a quick rise of the electronic temperature following the absorption of a pump pulse. For example, in the majority sub-band of $\mathrm{Fe}, 1.55 \mathrm{eV}$ photons are strongly absorbed by electrons from just below $E_{F}$ [6]. Owing to that, one can expect a significant contribution from this mechanism to the variations of the magneto-optical coefficient of Fe.

It is thus seen that even in the absence of electronic transport, when the laser excitation is homogeneous across the film thickness, the transient MOKE rotation and ellipticity exhibit different dynamics. This could potentially pose a problem for those experiments in which only one of these two quantities (for example, MOKE rotation) is registered. 


\section{Experimental results}

To illustrate the above considerations we now turn to the experimental data. We studied the transient MOKE response of three Fe samples, namely, thin (8 nm) and thick (34 $\mathrm{nm})$ films on top of a thick $(130 \mathrm{~nm})$ Au layer, and a thin $(8 \mathrm{~nm})$ Fe film grown on an insulating $\mathrm{MgO}$ substrate. The third $(\mathrm{Fe}-8 / \mathrm{MgO})$ sample represents the ultimate case where the impact of non-local contributions is minimized due to a nearly homogeneous excitation profile. The latter is illustrated with the red shaded area in Figure 1,b, as calculated using the $4 \times 4$ transfer matrix method [42].

For the pump-probe MOKE experiments we employed 14 fs-long, $1.55 \mathrm{eV}$ laser pulses (1 MHz repetition rate, $40 \mathrm{~mW}$ average power) split in a ratio of 4:1 into pump and probe beams incident on the sample at angles of 45 and 55 degrees, respectively. Using identical pump and probe photon energies, we intentionally enhance local state-filling effects which are of interest here. The ellipticity and rotation MOKE signal variations were detected by two home-built balanced detectors, whereas a broadband quarterwave plate was installed in front of one of them. This arrangement allowed for a simultaneous measurement of both transient MOKE rotation and ellipticity. The MOKE transients were measured in two saturating external magnetic fields of opposite polarities, directed in the plane of the sample in the longitudinal MOKE geometry. Then, the differences of the two datasets were taken to exclude the magnetization-independent contributions to the observed signals.

The experimental results are summarized in Figure 3. All transient variations are normalized to the static MOKE signals measured in the absence of the pump beam. All datasets shown here demonstrate a very quick, step-like effect at around zero time delay, that is, immediately after the arrival of the pump pulse. We argue that the shortest timescale visible in the data is determined by the time resolution of the experimental setup, which is about $20 \mathrm{fs}$. Considering the absence of transport effects in the current experimental conditions, these features are very unlikely to reflect real magnetization dynamics (see below) thus corroborating the assertion that the MOKE response alone at ultrashort time delays cannot be taken as a measure of the demagnetization process [24]. In what follows, we shall refer to these quasi-instantaneous variations of the MOKE signals as $\delta \theta(t=0)$, where $t=0$ indicates that the characteristic times of the relevant processes are shorter than our experimental resolution.

Moreover, it is clear that the transient MOKE rotation and ellipticity exhibit significantly different dynamics indicating the importance of the variations of the magneto-optical coefficients. Note that the huge relative variations of MOKE rotation at short time delays seen in Figure 3, a result from a very low static MOKE rotation for a 8 $\mathrm{nm}$-thin Fe film grown on $\mathrm{Au}$ (see inset). The significantly larger static MOKE ellipticity signal explains the absence of a similar effect in the respective MOKE ellipticity time trace.

Figure 3 illustrates that the particular sample structure greatly influences the apparent MOKE dynamics. The samples studied here exhibit very distinct transient 


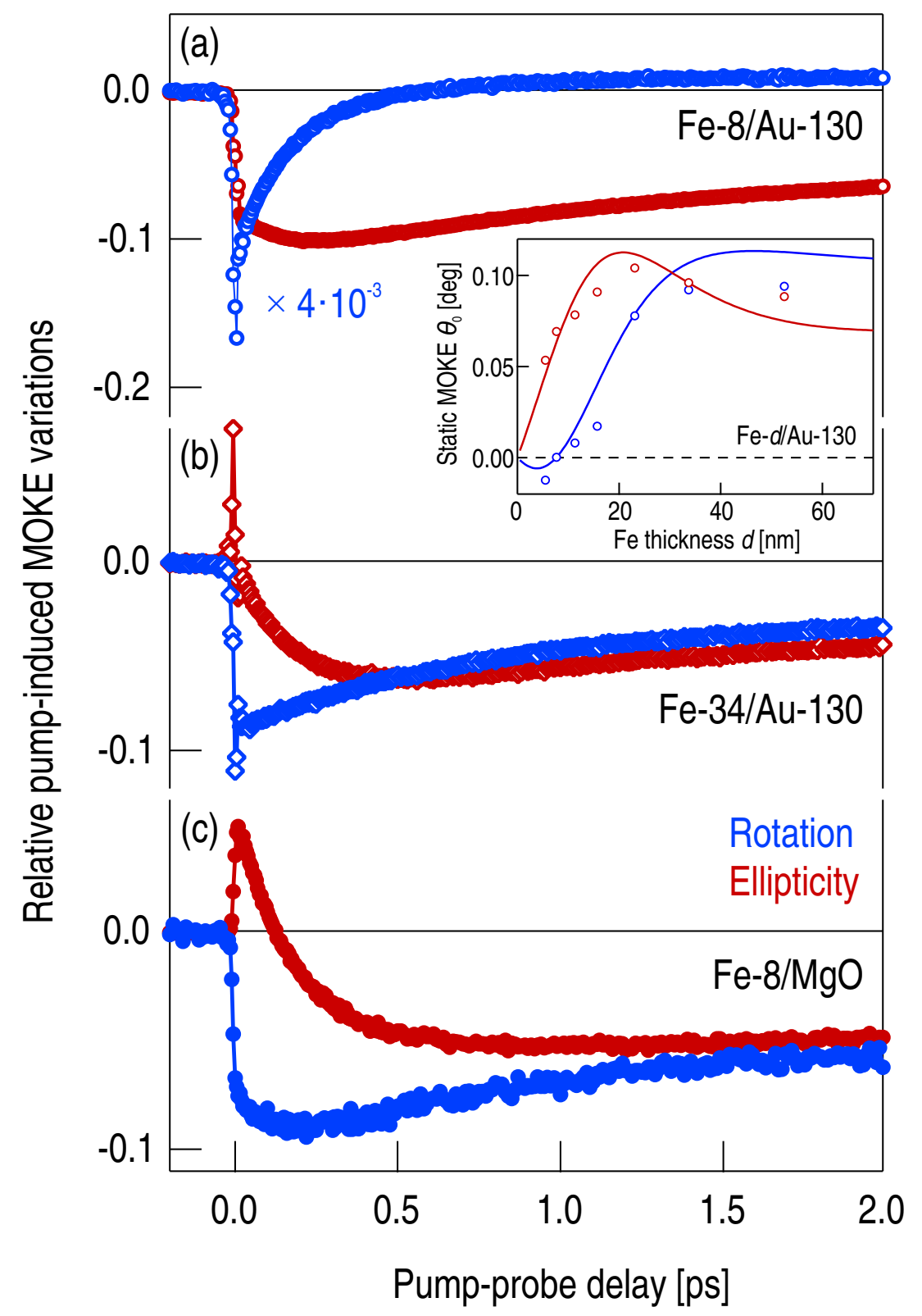

Figure 3. Experimental transient MOKE signals measured on (a) 8 nm-thin Fe film on $130 \mathrm{~nm} \mathrm{Au}$; (b) $34 \mathrm{~nm}$-thick Fe film on $130 \mathrm{~nm} \mathrm{Au}$; (c) $8 \mathrm{~nm}$-thin Fe film on insulating $\mathrm{MgO}$ substrate. Note the scaling factor $4 \times 10^{-3}$ for the MOKE rotation shown in panel (a). The inset in (a-b) shows both experimental (open symbols) and calculated (solid lines) values of the static MOKE signals $\theta_{0}$ as a function of Fe film thickness $d$.

MOKE signals with different timescales which are clearly seen in the MOKE rotation traces. We argue that the differences in the data shown here originate from the varying contributions related to the electronic transport. The local laser-induced demagnetization, however, can be studied in the Fe-8/MgO sample (Figure 3,c) where, as we have shown above, the non-local contributions are minimized. However, the difference in the MOKE rotation and ellipticity transients requires addressing the open 
question as to how they are related to the genuine magnetization dynamics.

\section{Towards genuine ultrafast demagnetization}

We further consider the complex MOKE angle in the form of $\Theta=\theta^{\prime}+i \theta^{\prime \prime}$, where $\theta^{\prime}$ and $\theta^{\prime \prime}$ account for the MOKE rotation and ellipticity, respectively. Because magnetization $M$ is real, this requires the magneto-optical coefficient to be complex as well: $\alpha=\alpha^{\prime}+i \alpha^{\prime \prime}=A \exp (i \varphi)$. It is worth noting that the variations of magnetization $\delta M(t)$ cannot change the phase of the MOKE response, and thus the variations of the latter should be understood as having an optical origin [28]. Indeed, since the magnetization $M$ in $\Theta=\alpha M$ is real, the phase of the complex value $\Theta$ is governed by $\alpha$ only. Assuming that the pump-induced effects are small, after the linearization Eq. 2 yields:

$$
\begin{aligned}
& \delta \theta^{\prime}=\delta M+\delta A-\kappa^{-1} \Delta \varphi \\
& \delta \theta^{\prime \prime}=\delta M+\delta A+\kappa \Delta \varphi
\end{aligned}
$$

where $\kappa=\theta_{0}^{\prime} / \theta_{0}^{\prime \prime}$ is the ratio between the static values of the MOKE rotation and ellipticity. It is immediately seen that if one of the static MOKE signals $\theta_{0}^{\prime}, \theta_{0}^{\prime \prime}$ is much stronger than the other, either $\kappa$ or $\kappa^{-1}$ becomes very large, and even small variations of the phase $\Delta \varphi$ completely dominate the pump-induced variations of the respective MOKE signal. This could lead to apparent variations in MOKE transients which are unrelated to the magnetization dynamics (cf. MOKE rotation in Fig. 3,a).

Equation 3 allows to identify the pump-induced phase dynamics:

$$
\Delta \varphi(t)=\frac{\kappa}{\kappa^{2}+1}\left(\delta \theta^{\prime \prime}(t)-\delta \theta^{\prime}(t)\right)
$$

The sum of the variations of the amplitude $\delta A$ and magnetisation $\delta M$ is, in turn, given by:

$$
\delta M(t)+\delta A(t) \equiv \delta g(t)=\frac{\delta \theta^{\prime}(t) \kappa^{2}+\delta \theta^{\prime \prime}(t)}{\kappa^{2}+1}
$$

The traces of $\delta g(t)$ and $\Delta \varphi(t)$ obtained for the $8 \mathrm{~nm}$-thin Fe film, where we found $\kappa \approx 1.57$, are shown in Figure 4 . It is seen that in our experiments, the phase variations are not larger than $5 \cdot 10^{-2}$ thus justifying the linearization procedure described above.

It is seen from Eq. 5 that the transient variations $\delta g(t)$ consist of magnetic $\delta M(t)$ and optical $\delta A(t)$ parts. We note that due to the conservation of angular momentum, light does not directly interact with spins other than via spin-orbit coupling [43, 44]. In practice, if the laser pulses are long enough $(>100 \mathrm{fs})$ it is difficult to distinguish the instantaneous dynamics from that with the characteristic timescale of the spin-orbit coupling. Governing the spin lifetimes, the latter was calculated to be on the order of 50-100 fs [20, 45]. In our case, 14 fs-long laser pulses allow for sufficient resolution to rule out quasi-instantaneous changes of magnetization upon laser irradiation and thus assume $\delta M(t=0)=0$. Here $t=0$ indicates, first of all, the pump-probe temporal overlap. 


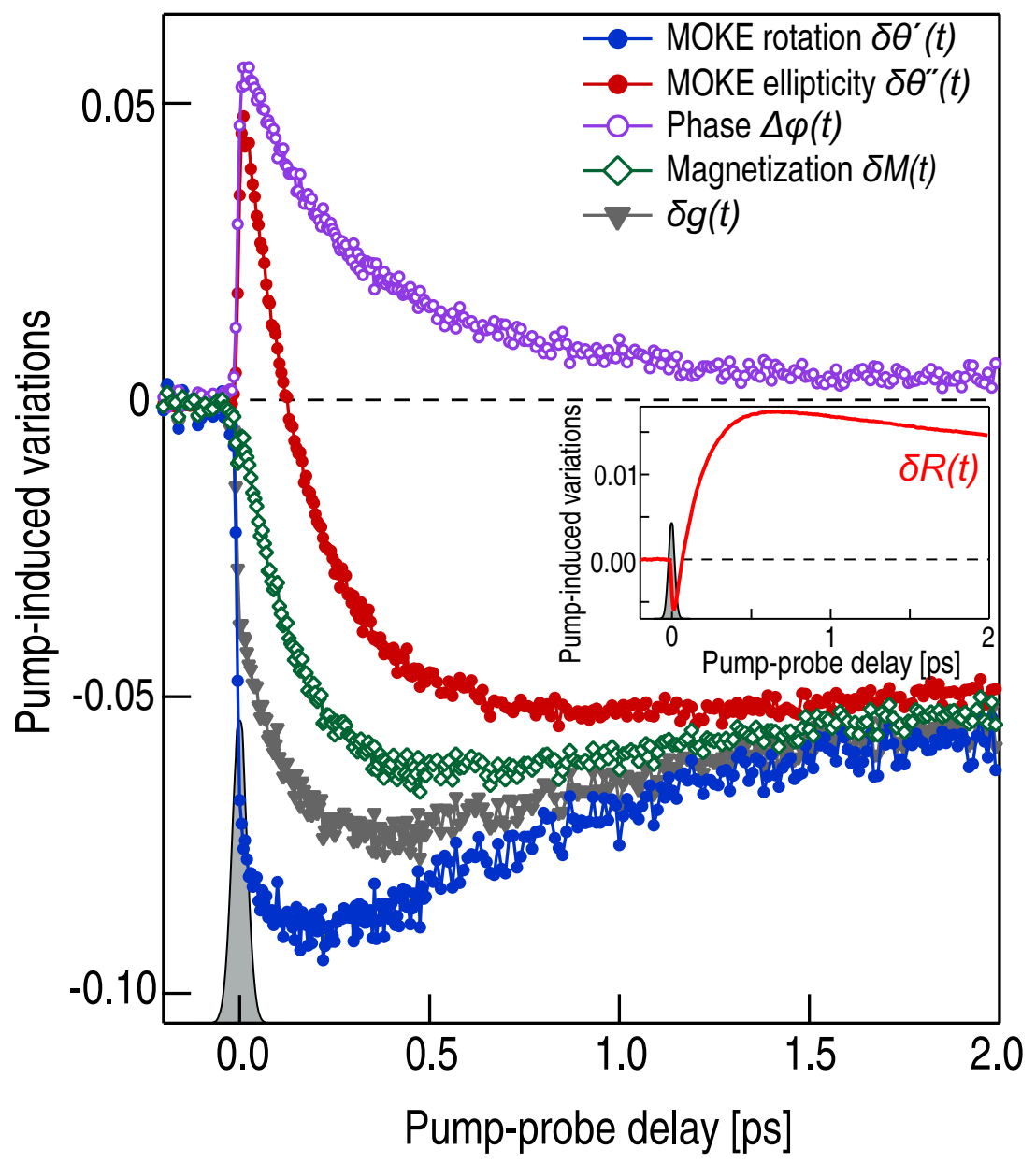

Figure 4. Retrieved pump-induced dynamics of Fe magnetization $\delta M(t)$ in $\mathrm{Fe}-8 / \mathrm{MgO}$ sample and variations of the phase of the magneto-optical coefficient $\Delta \varphi(t)$ obtained using Eq. 7. The gray triangles illustrate the transient variations $\delta g(t)$. Also shown are the transient MOKE signals $\delta \theta^{\prime}(t), \delta \theta^{\prime \prime}(t)$ obtained in the experiment (cf. Figure 3,c). Inset: Relative transient variations of the reflectivity $\delta R(t)$. The gray shaded areas represent the temporal profile of a 14 fs-long pump pulse.

Due to the finite pulse duration $t=0$ implicitly includes processes with characteristic times shorter than our experimental resolution. It is thus seen that the instantaneous variations of the MOKE signals $\delta \theta^{\prime}(0), \delta \theta^{\prime \prime}(0)$, as well as $\delta g(0)$ can only stem from the optical effects resulting in non-zero $\delta A(0), \Delta \varphi(0)$. In what follows, we assume that the optical part in $\delta g(t)$ given by the variations $\delta A(t)$ has the same origin and exhibits similar dynamics as the phase $\varphi(t)$ :

$$
\delta A(t)=\gamma \Delta \varphi(t) .
$$

Then the genuine magnetization dynamics can be retrieved:

$$
\delta M(t)=\delta g(t)-\frac{\delta g(t=0)}{\Delta \varphi(t=0)} \Delta \varphi(t)
$$


The transient magnetization variation $\delta M(t)$ is shown in Figure 4 along with the relative variations $\delta \theta^{\prime}(t)$ and $\delta \theta^{\prime \prime}(t)$ which are directly accessible in the experiment.

\section{Discussion}

We note that $a b$ initio calculations are highly desirable in order to verify to which extent Eq. 6 holds. However, in order to demonstrate the plausibility of this assumption, here we can analyse the two limiting cases. For example, consider two well-defined, isolated energy levels responsible for a single electronic transition which is driven by resonant laser excitation. It is clear that in this picture, the relaxation of both $A, \varphi$ is governed by the dynamics of the electronic population at these two energy levels and thus proceeds with similar time traces. Alternatively, consider a continuum of electronic states with numerous transitions excited by a broadband pump pulse. Despite each state in the continuum might have its own relaxation timescale, an effective evolution rate can be introduced (in the spirit of the central limit theorem). Due to the spectrally overlapping lineshapes of the individual transitions, one can again expect similar relaxation dynamics in both $A, \varphi$ with a corresponding effective rate. As such, in a real situation between these two limiting cases the assumption given by Eq. 6 should provide a reasonably close approximation to the relaxation dynamics.

Figure 4 summarizes the dynamics of all quantities discussed above including the observed MOKE signals. It illustrates that the rapid changes at very short time delays in both MOKE datasets $\delta \theta^{\prime}(t), \delta \theta^{\prime \prime}(t)$ are triggered by the ultrafast dynamics of magnetooptical coefficient $\alpha$. This suggests that the transients detected in the experiment are often determined by the temporal resolution of the experimental setup. For example, it can be misleading to evaluate the demagnetization rate from the MOKE rotation transients measured with 100 fs-long laser pulses. Our experiments performed with temporal resolution of 20 fs illustrate that both the rate and the magnitude of the ultrafast demagnetization are estimated inaccurately if only a single MOKE signal is taken into account. It is seen in Figure 4 that the demagnetization timescale in these experimental conditions is rather on the order of $200 \mathrm{fs}$, although a detailed analysis of this magnetization dynamics will be discussed elsewhere. Similar timescale of the local demagnetization processes can be expected in the two other samples (Figure 3,ab), although the total magnetization dynamics there is more complex. Transportrelated, non-local effects outlined in Figure 1 can introduce additional timescales into the observable MOKE time traces. Furthermore, redistribution of spins within the thick Fe film (Figure 3,b) modifies the MOKE response due to its in-depth sensitivity. For the assertions on the relative importance of these effects as compared to the local ones, $a b$ initio calculations as well as elaborated experimental methods are highly desirable.

Interestingly, the separation of the charge and spin contributions to the dynamics of the off-diagonal component of the dielectric tensor $\varepsilon_{i j}$ [28] on the ultrafast timescale is non-trivial. Indeed, while reflectivity is given by the diagonal components $\varepsilon_{i i}$, both $\varepsilon_{i i}$ and $\varepsilon_{i j}$ determine the magneto-optical coefficient $\alpha \propto \varepsilon_{i j} / \varepsilon_{i i}$. Because matrix 
elements of the electronic transitions which govern $\varepsilon_{i i}, \varepsilon_{i j}$ are not the same, the electronic contribution to the variations of these components exhibits, in general, different dynamics. As such, retrieving ultrafast dynamics of magnetization $M$ and magneto-optical coefficient $\alpha$ requires assumptions on the relaxation dynamics of the amplitude $\delta A(t)$ and phase $\Delta \varphi(t)$, as discussed above. This is illustrated in the inset of Figure 4 which demonstrates transient reflectivity changes $\delta R=\Delta R / R$ as measured simultaneously in our experimental setup. It is seen that $\delta R(t)$ exhibits clearly different dynamics to that of both $\delta \theta^{\prime}(t), \delta \theta^{\prime \prime}(t)$, and $\delta g(t)$. Resulting in a sizeable overestimation of the laser-induced demagnetization, our attempts to associate $\delta A(t)$ with $\delta R(t)$ rather than with $\Delta \varphi(t)$ (see Eq. 6) while maintaining $\delta M(t=0)=0$ corroborate our conclusion that the time-resolved reflectivity variations are of limited use for making assertions on ultrafast magnetization dynamics.

We note that the contribution of the variations of magneto-optical coefficients to the transient MOKE signals is emphasized in our experiments where the pump and probe beams have the same photon energy. This does not imply, however, that the twocolour pump-probe experiments, that is, where the pump and probe beams are of two distinct wavelengths $[11,19]$, are capable of producing MOKE data directly representing genuine magnetization dynamics. On the contrary, the heating-induced mechanism outlined in Figure 2,b does not depend on the exact path of the heating of the electronic subsystem. Furthermore, the contribution from primary HCs cannot be completely ruled out either, due to the possible engagement of the pump-repopulated electronic states in the interactions with the probe beam. The latter additionally highlights the importance of calculations of the electronic transitions involved in the light-matter interaction in each case.

\section{Conclusions}

To summarize, we have discussed the immense complexity of transient magneto-optical response if non-local, transport-related processes are admixed to the local ones. Careful experimental design could be employed in order to separate the two, thus allowing for a systematic study of genuine laser-induced demagnetization. Considering an example of such a system, a thin Fe film on an insulating substrate, we demonstrate further ambiguity of the experimentally obtained transient MOKE signals and their relation to the real magnetization dynamics. It is shown that full transient MOKE characterization including both rotation and ellipticity together with ultrashort (below $50 \mathrm{fs}$ ) temporal resolution is crucial for correct evaluation of ultrafast magnetization dynamics. We also propose a way to extract the latter from the experimental data and discuss the assumptions and limitations of the proposed algorithm. 


\section{Acknowledgements}

The authors are indebted to B. Rethfeld, M. Weinelt, H.C. Schneider, P. Oppeneer and K. Carva for fruitful discussions, as well as M. Wolf for his support. This work was partially funded by the Deutsche Forschungsgemeinschaft (ME 3570/1, SFB 616) and by the EU 7-th framework program (CRONOS).

\section{Referencing}

[1] Beaurepaire E, Merle J C, Daunois A and Bigot J Y 1996 Phys. Rev. Lett. 76 4250-4253

[2] Koopmans B, van Kampen M, Kohlhepp J T and de Jonge W J M 2000 Phys. Rev. Lett. 85 844-847

[3] Guidoni L, Beaurepaire E and Bigot J Y 2002 Phys. Rev. Lett. 89017401

[4] Battiato M, Carva K and Oppeneer P M 2010 Phys. Rev. Lett. 105027203

[5] Malinowski G, Longa F D, Rietjens J H H, Paluskar P V, Huijink R, Swagten H J M and Koopmans B 2008 Nature Phys. 4 855-858

[6] Melnikov A, Razdolski I, Wehling T O, Papaioannou E T, Roddatis V, Fumagalli P, Aktsipetrov O, Lichtenstein A I and Bovensiepen U 2011 Phys. Rev. Lett. 107076601

[7] Eschenlohr A, Battiato M, Maldonado P, Pontius N, Kachel T, Holldack K, Mitzner R, Föhlisch A, Oppeneer P M and Stamm C 2013 Nature Mater. 12 332-336

[8] Turgut E, La-o-vorakiat C, Shaw J M, Grychtol P, Nembach H T, Rudolf D, Adam R, Aeschlimann M, Schneider C M, Silva T J, Murnane M M, Kapteyn H C and Mathias S 2013 Phys. Rev. Lett. 110197201

[9] Rhie H S, Dürr H A and Eberhardt W 2003 Phys. Rev. Lett. 90247201

[10] Lisowski M, Loukakos P A, Melnikov A, Radu I, Ungureanu L, Wolf M and Bovensiepen U 2005 Phys. Rev. Lett. 95137402

[11] Cinchetti M, Albaneda M S, Hoffmann D, Roth T, Wüstenberg J P, Krauß M, Andreyev O, Schneider H C, Bauer M and Aeschlimann M 2006 Phys. Rev. Lett. 97177201

[12] Carley R, Döbrich K, Frietsch B, Gahl C, Teichmann M, Schwarzkopf O, Wernet P and Weinelt M 2012 Phys. Rev. Lett. 109057401

[13] Melnikov A, Prima-Garcia H, Lisowski M, Gießel T, Weber R, Schmidt R, Gahl C, Bulgakova N M, Bovensiepen U, and Weinelt M 2008 Phys. Rev. Lett. 100107202

[14] Stamm C, Kachel T, Pontius N, Mitzner R, Quast T, Holldack K, Khan S, Lupulescu C, Aziz E F, Wietstruk M, Dürr H A and Eberhardt W 2007 Nature Mater. 6 740-743

[15] Boeglin C, Beaurepaire E, Halte V, Lopez-Flores V, Stamm C, Pontius N, Dürr H A and Bigot J-Y 2010 Nature (London) 465 458-461

[16] Wietstruk M, Melnikov A, Stamm C, Kachel T, Pontius N, Sultan M, Gahl C, Weinelt M, Dürr H A and Bovensiepen U 2011 Phys. Rev. Lett. 106127401

[17] Radu I, Vahaplar K, Stamm C, Kachel T, Pontius N, Dürr H A, Ostler T A, Barker J, Evans R F L, Chantrell R W, Tsukamoto A, Itoh A, Kirilyuk A, Rasing Th and Kimel A V 2007 Nature (London). 472 205-208

[18] Bergeard N, Lopez-Flores V, Halte V, Hehn M, Stamm C, Pontius N, Beaurepaire E and Boeglin C 2014 Nature Comm. 53466

[19] Koopmans B, Malinowski G, Longa F D, Steiauf D, Fähnle M, Roth T, Cinchetti M and Aeschlimann M 2010 Nature Mater. 9 259-265

[20] Carva K, Battiato M, Legut D and Oppeneer P M 2013 Phys. Rev. B 87184425

[21] Zhang G P and Hübner W 2000 Phys. Rev. Lett. 85 3025-3028

[22] Krieger K, Dewhurst J K, Elliott P, Sharma S and Gross E K U 2015 J. Chem. Theor. Comp. 11 4870-4874

[23] Töws W and Pastor G 2015 Phys. Rev. Lett. 115217204 
[24] Oppeneer P M and Liebsch A 2004 J. Phys.: Condens. Matter 16 5519-5530

[25] Lisowski M, Loukakos P, Bovensiepen U, Stähler J, Gahl C and Wolf M 2004 Appl. Phys. A 78 165-176

[26] Bovensiepen U 2007 J. Phys. Cond. Mat. 19083201

[27] Hohlfeld J, Matthias E, Knorren R and Bennemann K H 1997 Phys. Rev. Lett 78 4861-4864

[28] Carpene E, Boschini F, Hedayat H, Piovera C, Dallera C, Puppin E, Mansurova M, Münzenberg M, Zhang X and Gupta A 2013 Phys. Rev. B 87174437

[29] Carpene E, Hedayat H, Boschini F and Dallera C 2015 Phys. Rev. B 91174414

[30] Wieczorek J, Eschenlohr A, Weidtmann B, Rösner M, Bergeard N, Tarasevitch A, Wehling T O and Bovensiepen U 2015 Phys. Rev. B 92174410

[31] Hohlfeld J, Wellershoff S S, Güdde J, Conrad U, Jähnke V and Matthias E 2000 Chem. Phys. 251 $237-258$

[32] Walowski J and Münzenberg M 2016 J. Appl. Phys 120140901

[33] Koopmans B, Ruigrok J J M, Longa F D, and de Jonge W J M 2005 Phys. Rev. B 95267207

[34] Battiato M, Carva K and Oppeneer P M 2012 Phys. Rev. B 86024404

[35] Schellekens A J, Verhoeven W, Vader T N and Koopmans B 2013 Appl. Phys. Lett. 102252408

[36] Zak J, Moog E R, Liu C and Bader S D 1990 J. Magn. Magn. Mater. 89 107-123

[37] Hamrle J, Pistora J, Hillebrands B, Lenk B and Münzenberg M 2010 J. Phys. D: Appl. Phys. 43 325004

[38] Argyres P N 1955 Phys. Rev. 97 334-345

[39] Bennett H S and Stern E A 1965 Phys. Rev. 137 448-461

[40] Kampfrath T, Ulbrich R G, Leuenberger F, Münzenberg M, Sass B and Felsch W 2002 Phys. Rev. B 65104429

[41] Moruzzi V L, Janak J F and Williams A R 1978 Calculated electronic properties of metals 1st ed (New York: Pergamon Press Inc.)

[42] Born M and Wolf E 1964 Principles of optics: electromagnetic theory of propagation, interference and diffraction of light (Oxford: Pergamon Press)

[43] Shen Y R and Bloembergen N 1966 Phys. Rev. 143 372-384

[44] Kimel A V, Kirilyuk A and Rasing T 2007 Las. Phot. Rev. 1 275-287

[45] Kaltenborn S and Schneider H C 2014 Phys. Rev. B $90201104(\mathrm{R})$ 